\title{
«Le précieux livre de W. Bölsche»
}

Freud et la culture évolutionniste allemande du début du XX $X^{e}$ siècle*

\author{
Rémy Amouroux
}

\section{Summary}

Wilhelm Bölsche (1861-1939) is the author of a poetic history of the evolution of love entitled Das Liebesleben in der Natur (1898-1903). This work, inspired by the writings of biologist Ernst Haeckel, was greatly successful in Germany at the beginning of the 20th century. Freud kept a copy of the three volumes in his London library and cites the text in his lectures on an Introduction to psychoanalysis.

Bölsche develops an Entwicklungsgeschichte (history of evolution) of the distinguishing sexuality of several types of love (oral, anal and urinary). In addition, he describes the "zoological reactionary" homosexual and ties this sexual behaviour to the history of the development of anal sexuality. This paper will address an excerpt on this topic from Bölsche's text that has been translated for the occasion. The task at hand is to prepare the ground for a study of German evolutionism, both popular and scientific, and its ties to psychoanalysis.

\footnotetext{
* Le présent article est le prolongement de mon mémoire de DEA, «Freud, Lamarck et le lamarckisme», soutenu à l'Ecole des Hautes Etudes en Sciences Sociales (Paris), en juin 2002 auprès de Mme Jacqueline Carroy et de M. Pietro Corsi.

Mes plus vifs remerciements à Jacqueline Carroy, Directrice d'études à l'Ecole des Hautes Etudes en Sciences Sociales (Paris), mon directeur de thèse, ainsi qu'à Marielène Weber, traductrice de l'allemand, qui a eu l'obligeance d'établir la traduction française définitive de l'extrait présenté de Wilhelm Bölsche.
}

Rémy Amouroux, Hôpital Armand Trousseau, Service d'anesthésie, 26, avenue du Docteur Arnold-Netter, F-75571 Paris cedex 12 (remy.amouroux@laposte.net). 


\section{Résumé}

Wilhelm Bölsche (1861-1939), est l'auteur d'une histoire poétique de l'évolution de l'amour: Das Liebesleben in der Natur (1898-1903). Ce texte, singulier et lyrique, qui s'inspire des travaux du biologiste Ernst Haeckel, connaîtra un vif succès au début du $\mathrm{XX}^{\mathrm{e}}$ en Allemagne. Freud, qui possédait un exemplaire des trois volumes de ce texte dans sa bibliothèque de Londres, le cite dans ses conférences d'Introduction à la psychanalyse. Bölsche, dans ce texte, développe une Entwicklungsgeschichte (histoire de l'évolution) de la sexualité qui distingue plusieurs formes d'amour (oral, anal et urinaire). Il qualifie, en outre, l'homosexuel de «réactionnaire zoologique», et lie ce comportement sexuel à l'histoire du développement de la sexualité anale. Nous commenterons, à ce sujet, un extrait de ce texte, traduit pour l'occasion. L'enjeu sera de poser les jalons d'une étude de l'évolutionnisme allemand, tant populaire que scientifique, et de ses liens avec la psychanalyse.

A partir de 1885, date à laquelle il fut nommé Privatdozent, Freud fit des cours à l'université de Vienne. Il ne rédigea aucun de ces cours, à l'exception de la série donnée durant les semestres d'hiver de 1915/16 et de 1916/17. Cette trentaine de cours fut publiée presque aussitôt sous le titre Vorlesungen zur Einführung in die Psychoanalyse (Conférences d'introduction à la psychanalyse $)^{1}$. Dans cette présentation de la psychanalyse, qui s'adressait à des auditeurs venant de toutes les facultés, Freud aborde la question du moi et de son développement en la mettant en parallèle avec ce qu'il appelle la libido et le développement de celle-ci:

Tous deux sont au fond des legs, des répétitions abrégées de l'évolution que l'humanité dans son ensemble a parcourue depuis la nuit des temps. Pour ce qui est du développement de la libido, il me semble qu'on voit tout de suite cette origine phylogénétique. Rappelez-vous, dans telle classe d'animaux l'appareil génital a des rapports très étroits avec la bouche, dans telle autre il est inséparable de l'appareil d'excrétion, et dans telle autre encore il est rattaché aux organes de la locomotion. Vous trouverez cela, décrit de façon attrayante, dans le précieux livre de W. Bölsche. ${ }^{2}$

Cette référence prouve la réelle popularité, à l'époque, du livre phare du polygraphe allemand Wilhelm Bölsche: une grande «histoire de l'évolution de l'amour» en trois volumes, Das Liebesleben in der Natur. Eine Entwicklungsgeschichte der Liebe $e^{3}$, dont la publication s'échelonna de 1898 à 1903.

1 Freud 1922.

2 Freud 1922, 334.

3 Bölsche, 1898-1903. 


\section{Un auteur populaire du tournant du siècle}

Né à Cologne en 1861, dans une famille bourgeoise et intellectuelle, Wilhelm Bölsche est éduqué dans le libéralisme protestant. Son père, Karl Bölsche, rédacteur en chef du journal de Cologne, Kölnische Zeitung, avait commencé par faire des études de théologie mais renonça à la carrière pastorale. Il s'était rangé du côté des partisans du théologien David Friedrich Strauss, auteur d'une histoire de la vie de Jésus, publiée en 1835, qui soumet les évangiles à la critique historique.

Après un parcours scolaire médiocre, il quitte l'école sans même obtenir l'équivalent allemand du baccalauréat, l'Abitur. Il entame alors un voyage à travers l'Europe, et va suivre des cours de philosophie et d'histoire de l'art à Bonn et à Paris. Par la suite, il s'établit à Berlin, où il rencontre le romancier, philosophe et théologien libéral Bruno Wille. Bölsche va mêler sa passion pour la littérature et la poésie avec celle qu'il voue aux sciences naturelles. Ainsi tente-t-il de tirer des sciences naturelles une méthode applicable au domaine esthétique dans son livre, «Les sciences naturelles, fondement de la poésie: prolégomènes à une esthétique réaliste» ${ }^{4}$ publié en 1887. Les deux volumes de son «Histoire du développement de la nature», Entwicklungsgeschichte der Natur ${ }^{5}$, publiés en 1894 et en 1896, attirèrent sur lui l'attention du public. L'écrivain est contacté par le futur éditeur à succès de Leipzig, Eugen Diederichs (1867-1930). Ce dernier recherche des auteurs pour sa nouvelle maison d'édition, qui entend promouvoir des ouvrages qui mêlent poésie et science. De 1898 à 1903, Bölsche publie chez Diederichs les trois volumes de Das Liebesleben in der Natur. Eine Entwicklungsgeschichte der Liebe, dont le succès est tel, 80000 exemplaires en 1927, que l'ouvrage est réédité quatre fois en l'espace de dix ans. Parallèlement, Bölsche collabore activement à la revue à grand tirage, Kosmos. Tout au long de sa vie, il accumule les ouvrages: des essais - par exemple «Nature et art», Natur und Kunst, en deux volumes publiés à Leipzig en 1921-1923, et, «Les conversations sur les sciences naturelles et la culture: Meurs et deviens», Stirb und Werde. Naturwissenschaftliche und kulturelle Plaudereien, publié à Jena en 1921 -, mais aussi des romans, par exemple le roman historique, Paulus. Roman aus der Zeit Kaiser Marcus Aurelius (Paul, un roman de l'époque de l'empereur Marc Aurèle), publié en 1885; le roman humoristique, Der Zauber des Königs Arpus (L'envoûtement du roi Arpus), publié deux après, et constamment réédité durant les décennies suivantes; le roman autobio- 
graphique, Die Mittagsgöttin (La déesse de midi), publié en 1910. Il entretient enfin une importante correspondance avec de nombreuses personnalités littéraires et scientifiques, dont le biologiste Ernst Haeckel et la romancière et psychanalyste Lou Andreas-Salomé. Wilhelm Bölsche meurt en 1939.

Dans son ouvrage The Descent of Darwin ${ }^{6}$, qui traite de la vulgarisation du darwinisme en Allemagne entre 1860 et 1914, l'historien des sciences américain Alfred Kelly soutient que Bölsche a été oublié de l'histoire de la diffusion des théories évolutionnistes, alors que, selon lui, «Bölsche fut probablement de tous les auteurs d'ouvrages de langue allemande qui n'étaient pas des fictions, le plus vendu avant $1933 »^{7}$. Kelly affirme même qu'il était le vulgarisateur scientifique par excellence pour ses contemporains, et ce, devant Haeckel. Ainsi, La descendance de l'homme de Bölsche, publiée tout d'abord en feuilleton dans la revue à grand tirage Kosmos, sera ensuite diffusée en librairie sous forme d'ouvrage, qui s'arracha à plus de 100000 exemplaires $^{8}$. Mais Bölsche, comme nous le verrons, c'est aussi un style singulier: dès 1907, le critique viennois Egon Friedell qualifiait de «Bölschiade» ce type de vulgarisation scientifique aux accents étonnamment lyriques 9 . Enfin, Ellenberger, dans son célèbre ouvrage, nous donne une interprétation intéressante de ce type de littérature:

Contrairement à la légende assez répandue de nos jours qui voudrait nous faire croire que cette époque [celle de Freud] se caractérisait par un obscurantisme sexuel, il n'existait alors, du moins sur le continent européen, aucune entrave à la publication, à la diffusion et à la lecture de ces ouvrages; c'est à cette époque qu'apparurent un peu partout des ouvrages populaires sur la sexualité. En Allemagne, par exemple, un livre de Bölsche, la vie amoureuse dans la nature, $[\ldots]$ fut un succès de librairie..$^{10}$

\section{Le roman de l'évolution selon Wilhelm Bölsche}

Das Liebesleben in der Natur propose une histoire de l'amour qui est celle de l'évolution de l'organisation sexuelle de l'ensemble des êtres vivants. L'ouvrage peint, par conséquent, comme l'indique Freud, l'évolution de la reproduction sexuelle depuis les organismes les plus primitifs jusqu'à l'homme, avec en filigrane la loi biogénétique de Haeckel, selon laquelle «l'ontogenèse récapitule la phylogenèse», c'est-à-dire que l'évolution de l'individu serait en quelque sorte la succession des périodes vécues par ses

\footnotetext{
6 Kelly 1981.

7 Kelly 1981, 37.

8 Daum 1997.

9 Fellman 1988.

10 Ellenberger 1994, 323.
} 
ancêtres ${ }^{11}$. Les pages présentées ci-dessous sont extraites du chapitre intitulé «La porte de l'amour»(Die Liebespforte). Nous les avons choisies parce qu'elles sont caractéristiques de «la description attrayante» des rapports possibles de l'appareil génital avec d'autres organes, dont Freud créditait Bölsche. Ce dernier y retrace, en effet, une histoire phylogénétique de l'anatomie sexuelle, où il distingue plusieurs stades, ou phases anatomiques, corrélatives de certaines formes d'amour ou de sexualité.

\section{Une histoire des orifices corporels}

Tu te souviens que plus haut nous est apparue fugitivement la possibilité d'un amour anal, d'un vrai amour passant par l'orifice anal.

Lorsque les matières sexuelles se détachaient encore de la paroi stomacale et que l'estomac fut ensuite devenu un tube qui comportait une ouverture antérieure et une ouverture postérieure, l'anus pouvait naturellement être utilisé, tout comme la bouche, pour l'évacuation du sperme et des ovules. Mais, comme tu le sais, cet amour stomacal cesse très tôt de jouer le moindre rôle dans ton ascendance, car les organes génitaux s'installent dans la cavité abdominale et utilisent toujours le rein comme voie des sortie. Cela commence déjà chez les vers et s'étend au règne de tes ancêtres directs, celui des animaux vertébrés. Cependant, au cours de ce mouvement ascendant, tu vois également se manifester une tendance qui, du moins après coup, crée de nouveau un lien entre le canal intestinal et l'amour.

Cela commence par un tour de force de celui qui occupe le degré le plus bas dans la hiérarchie des vertébrés, le fameux amphioxus qui se trouve encore à la frontière entre le ver et le poisson. Chez lui, reins et matières sexuelles débouchent déjà bel et bien dans une cavité unique, qui, bien qu'elle ait encore d'autres fonctions, peut aussi être qualifiée de canal urinaire. Ce canal commun, cependant, ouvre par des fentes sur la partie antérieure des intestins, et en passant à travers celles-ci, les ovules de la femelle, de même que les spermatozoïdes du mâle, finissent par sortir joyeusement de la vraie bouche de l'amphioxus. Si cet état des choses avait continué à s'implanter, cet amour urinaire aurait fini par redevenir malgré tout un amour oral, et tu devrais aujourd'hui faire transiter par le gosier, outre l'air et la nourriture, l'urine, le sperme et les enfants. Mais les multiples raisons qui étaient en défaveur de tout amour oral, étaient visiblement encore deux fois plus fortes après que, de surcroît, l'amour et l'urine furent réunis. Voilà pourquoi monsieur Amphioxus est le premier et le dernier vertébré qui aime avec la bouche. [...]

Chez un grand nombre de poissons, les ovules, le sperme, l'urine et les excréments solides s'écoulent tous ensemble par un seul et unique orifice. C'est en particulier le cas chez tes ancêtres plus directs, les requins. L'amphibien, c'est-à-dire la grenouille et le triton, ne connaissent plus rien d'autre. Tous les canaux du corps, qui sont orientés vers l'arrière, convergent en un point unique: le gros rectum. [...]

Si tu voulais trouver une appellation pour ce nouvel état des choses, il te faudrait en créer une qui engloberait l'amour urinaire et l'amour anal. Le naturaliste te propose un terme, même s'il n'est pas très bon. Il appelle une telle réunion du méat urinaire et de l'anus, chaque fois qu'il la rencontre chez des animaux, tout simplement «cloaque». Et nous en serions donc arrivés à l'amour cloacal. Les animaux appartenant à ce stade, qui, à la manière des corégones, s'aiment en rapprochant le plus possible leurs orifices d'évacuation du sperme et des ovules, du fait de cet état des choses devaient, dans l'acte d'amour, tout simplement tourner leurs «cloaques» l'un vers l'autre.

11 Pour plus de précision sur la loi biogénétique de Ernst Haeckel, cf. Mengal 1993. Sur la question de l'importance de Ernst Haeckel et de sa loi en psychanalyse, cf. Ritvo 1992 et Sulloway 1992. 
Depuis le triton, cet amour cloacal a effectivement joué un rôle décisif pendant longtemps encore. Tous les reptiles l'ont hérité de lui, les lézards, les serpents, les crocodiles et les tortues, et en tout cas aussi tous les monstres reptiles disparus, les ichtyosaures, les chiroptères, les terribles dragons, etc. Quant à l'oiseau, ce n'est rien d'autre qu'un lézard volant très développé, à sang chaud et recouvert de plumes. C'est pourquoi, invariablement, tu trouves développé, chez la poule et chez l'autruche, chez l'aigle et chez le rossignol, comme chez les milliers d'autres êtres ailés des champs, de la forêt, de la mer et des airs, un vrai cloaque - l'œuf solide et la semence liquide, l'excrément de l'intestin et l'urine des reins (chez eux, semblable à de la bouillie) sortant toujours par la même grande porte postérieure du corps.

Et ce n'est que chez le mammifère que, par la suite, se produit de nouveau un grand arrêt. Car si grand qu'ait été le triomphe de la parcimonie qui avait réussi à fondre tous les orifices en un seul, il a été impossible de s'y tenir jusque dans le degré le plus élevé de l'évolution. Encore aujourd'hui vit en Australie, un animal, l'ornithorynque, qui incarne le changement qui mène au sommet radieux de toute la nature animée de la terre. L'ornithorynque pond encore des œufs, à peu près comme le fait un oiseau, ou plutôt un lézard. De plus, l'ornithorynque a vraiment encore un cloaque, et il est le seul des mammifères à en avoir un. Car c'est sans aucun doute déjà un mammifère puisqu'il nourrit ses petits avec du lait. Mais il a tout de même encore un cloaque. [...]

Puis vient le marsupial - la sarigue et le kangourou -, et c'est la fin du cloaque. Tout à coup, il y a de nouveau un solide verrou, une cloison, entre l'ouverture urinaire et sexuelle et l'ouverture anale. A partir de ce moment-là, il y a tout de même de nouveau deux orifices. Et cela reste ainsi jusqu'à toi, qui l'as finalement hérité des singes. L'amour cloacal, tu ne le connais plus, sauf dans le cas d'un acte violent particulièrement malheureux, c'est-à-dire si, au moment de l'accouchement, la paroi située vers l'anus se déchire, mais ce n'est plus quelque chose de normal; c'est un cas pathologique isolé.

En tous cas, il serait totalement inutile d'essayer de faire passer de nouveau la procréation par l'anus. Depuis l'ornithorynque, aucun spermatozoïde de mammifère n'est plus jamais arrivé par l'extrémité postérieure de l'intestin jusqu'à une ovule. Tout au plus peut-on voir dans l'acte pédérastique une caricature d'essai externe.

Le pédéraste, en recherchant la voie intestinale comme voie sexuelle, régresse dans une certaine mesure jusqu'à l'ornithorynque, sauf que ce qui était conforme à la nature chez ce dernier et en possédait le sens plein et sacré se transforme en absurdité dans son cas. Il est vrai que la tentative simultanée de constituer un individu aimant composé de deux hommes est une régression encore plus grande. Elle fait redescendre jusqu'à ces vers et ces poissons hermaphrodites, chez qui chaque moitié sexuée était encore à la fois mâle et femelle, c'està-dire qu'un organe mâle pouvait féconder dans un corps qui possédait lui-même des organes mâles.

Du reste, les racines tenaces de la pédérastie, qui se manifeste tout au long du laborieux progrès de l'humanité, sont très enchevêtrées, allant bien au-delà de ces réminiscences, et seul un observateur très superficiel prétendra se débarrasser de la question en invoquant une seule résonance. Si sur un navire isolé en mer, le marin, privé de toute compagnie féminine, a recours à la pédérastie, la racine est incontestablement différente: l'acte de pédérastie n'est qu'une variante de l'onanisme, un pis-aller vu le manque de femmes. Et il est hors de doute que depuis les temps les plus reculés c'est une des situations qui en toute logique n'a cessé de l'engendrer. Mais si tu trouves des motifs pédérastiques dans l'art le plus élevé, dans le monde des idées imprégné d'art de la Grèce antique, dans l'idéal incarné par Antinoüs, dans la poésie orientale, et encore et toujours à la Renaissance et dans les Temps modernes, cela te mènera à une racine d'une toute autre nature. La pédérastie, dans ce cas, a son origine dans un acte en lui-même parfaitement moral: c'est d'abord de l'amour à distance. L'œil prend plaisir à la beauté du corps masculin, comme à celle du corps féminin, et cette beauté lui fait goûter la sensualité supérieure, spiritualisée, de l'amour à distance. Mais, par la suite, les motifs de cet amour à distance se brouillent: il devient un essai d'amourfusion, ce qui signifie tomber du sublime dans une situation, dont la punition la plus dure consiste incontestablement dans le ridicule. On peut encore mettre en évidence d'autres voies qui mènent à la pédérastie. En tous cas, ce qui est intéressant, y compris par rapport à l'amphioxus et à l'ornithorynque, c'est le «réactionnaire zoologique», qu'il y a dans le pédé- 
raste comme dans celui qui se masturbe. Quant au caractère tragi-comique qui est le sien, c'est un trait commun à tous les réactionnaires. ${ }^{12}$

Ce texte peut sembler fort étrange pour un lecteur du début du XXI ${ }^{\mathrm{e}}$ siècle. Comme nous l'avons déjà signalé, Bölsche, loin d'être marginal, constitue l'auteur vulgarisateur phare du début du XX $\mathrm{X}^{\mathrm{e}}$ siècle en Allemagne. A ce titre, la récente publication de la correspondance entre Bölsche et Haeckel par Rosemarie Nöthlich, nous permet de rapporter les propos de Haeckel au sujet de Das Liebesleben in der Natur:

Je dois vous adresser mes sincères remerciements pour votre livre esthético-biologique sur l'amour dans la nature. Je l'ai lu aussitôt, d'un bout à l'autre, avec le plus grand intérêt, puisque j'avais moi-même le projet, depuis plusieurs années, d'écrire quelque chose de semblable. Vous l'avez fait d'une bien meilleure façon que je n'aurais pu le faire, en particulier au sujet de la forme légère et élégamment artistique. ${ }^{13}$

Parmi les particularités stylistiques de l'«Histoire de l'évolution de l'amour», il est intéressant de relever que Bölsche tutoie son lecteur, et propose un ton à la fois familier et emphatique. L'histoire de l'évolution devient un récit, qui prend par moments l'allure d'un conte aux rebondissements divers, et des expressions imagées, parfois cocasses, animent les descriptions. La «porte de l'amour» et toutes les autres «portes» du corps sont personnifiées et se voient attribuer, l'anus comme la bouche, des sentiments, ou quelque volonté secrète, que seule la Nature connaît. Le monde humain est totalement intégré au monde naturel: «l'acte pédérastique», est comparé à la sexualité de l'ornithorynque, comme à celle d'un animal marin primitif, l'amphioxus. De même, les références à l'art antique, à l'idéal incarné par Antinoüs croisent celles au cloaque primitif des reptiliens.

Comme Freud, Bölsche, qui emploie les mêmes termes que celui-ci, pour les diverses formes d'amour ou de sexualité (oral, anal et urinaire ou urogénital), lie l'homosexualité avec un retour à l'amour anal. La diversification des orifices corporels (bouche, cloaque, anus et appareil uro-génital) est présentée comme corrélative de différentes formes de sexualité: l'amour oral (Mundliebe), dont le principal représentant serait l'amphioxus, chez qui la bouche joue un rôle primordial dans la reproduction; l'amour anal (Afterliebe) où l'ornithorynque s'illustre par son anatomie qualifiée de «réactionnaire»; l'amour urinaire (Urinliebe), qui apparaît avec les marsupiaux et que l'on retrouve chez tous les mammifères jusqu'à l'homme.

Bölsche compare le comportement homosexuel humain aux pratiques sexuelles et à l'anatomie de l'ornithorynque. Il considère ce mammifère comme un «réactionnaire zoologique». L'expression est tout à fait intéres-

12 Bölsche 1898-1903, 255-261.

13 Nöthlich 2002, 85. 
sante. Rappelons que l'ornithorynque constitue un animal particulier pour les zoologues. Sa découverte arriva comme un défi. Dès 1802, l'année même de sa description anatomique détaillée par un certain Sir Everard Home, il est inclus dans la classe des mammifères, dans laquelle un nouvel ordre est créé, désigné sous le nom de monotrème. Ce terme, qui signifie «un seul orifice», fait allusion à la présence d'un cloaque, alors qu'anus et orifice génito-urinaire sont séparés dans les autres ordres de mammifères. Bölsche semble donc voir chez l'ornithorynque une sorte de tentative de retour à une phase antérieure de l'évolution des animaux. Ce dernier possède, en effet, un cloaque, ce qui est pourtant une caractéristique des reptiliens.

\section{De la théorie des stades à la fantaisie phylogénétique: Freud lecteur de Bölsche?}

Nous l'avons dit, Freud ne cite Bölsche qu'une seule fois dans son œuvre scientifique. Dès lors, on peut se demander quelle serait la légitimité de l'hypothèse qui ferait de Freud un lecteur de Bölsche? Sur cette question précise nous avons plusieurs éléments de réponse. Bölsche est certes, pour ses contemporains, un vulgarisateur de renom, mais reste à connaître et à préciser sa réception dans les milieux psychiatriques et psychanalytiques. Quelle a pu être la place de Bölsche parmi les sexologues? Il semblerait que ni le psychiatre Richard von Krafft-Ebing, ni le neurologue Albert Moll ne l'aient jamais cité. Le caractère vulgarisateur et non académique de Bölsche pourrait expliquer cela, comme l'illustre cette remarque du psychiatre et entomologiste suisse Auguste Forel:

Un auteur allemand, ed. Bölsche (Das Liebesleben in der Natur. La vie de l'amour dans la nature), a décrit dernièrement l'amour dans les êtres organisés, sans oublier l'homme, sur un ton de plaisanterie forcé qui gâte les connaissances approfondies de l'auteur sur les sujets zoologiques et autres qu'il traite. ${ }^{14}$

On retrouve peu de références à Bölsche dans les textes de psychanalystes avant l'allusion de Freud en 1917 à Das Liebesleben in der Natur dans les conférences d'Introduction à la psychanalyse. Cependant, en 1908 lors d'une des réunions du mercredi des premiers analystes à Vienne, l'écrivain et philosophe Wittels, qui prendra en 1910 la voie de la dissidence, fait une conférence où les idées de Bölsche seront évoquées ${ }^{15}$. Mais c'est chez Sandor Ferenczi que l'on trouve les références les plus nombreuses à Bölsche. On peut citer en particulier son Thalassa, dont le thème, la version psychana-

15 Federn/Nünberg 1978, 360-367. 
lytique des origines de la vie sexuelle, n'est pas sans rappeler notre propos. Au sujet du rapport en ontogenèse et phylogenèse, il y écrit:

Des idées semblables à celles que nous venons d'exprimer nous en retrouvons - sous forme de comparaisons et d'images poétiques - dans les écrits pleins d'imagination et d'esprit de Bölsche, auteur populaire bien connu qui n'est pourtant pas encore apprécié à sa juste valeur comme penseur original. ${ }^{16}$

Enfin, Freud possédait, dans sa bibliothèque de Londres, un exemplaire de l'édition princeps du deuxième et du troisième volume (1900 et 1903) de Das Liebesleben in der Natur, ainsi qu'un exemplaire portant la mention «Freud 22. XI. 09» de l'édition de 1909 du premier volume ${ }^{17}$.

Nous allons maintenant essayer de cerner les éléments qui permettent de créditer la thèse d'une certaine forme de réappropriation freudienne des «Entwicklungsgeschichten» (histoires de l'évolution) à la Bölsche. Rappelons qu'il ne s'agit ici en aucun cas de faire de Bölsche un précurseur de Freud, mais plutôt de souligner l'importance de la référence évolutionniste dans certains textes psychanalytiques. Nous nous appuierons sur deux textes qui possèdent un statut différent dans le corpus psychanalytique: un texte fondateur avec les Trois essais sur la théorie sexuelle (1905) $)^{18}$, et un texte plus controversé, la Vue d'ensemble sur les névroses de transfert $(1915)^{19}$.

Parmi les écrits de Freud, Trois essais sur la théorie sexuelle reste un écrit fondamental et incontournable pour les psychanalystes. Je m'attacherai ici surtout à la troisième édition, celle de 1915. C'est en effet à cette époque que Freud émet l'idée d'une organisation prégénitale de la libido, et introduit les expressions «organisation sexuelle orale» et «organisation sexuelle anale».

La notion d'érotisme anal apparaît dès 1908 dans un article intitulé, «Caractère et érotisme anal $»^{20}$. Freud y met en relation des traits de caractère de l'adulte et l'érotisme anal de l'enfant. Il faut, cependant, attendre l'article de 1913, «La disposition à la névrose obsessionnelle» ${ }^{21}$, pour que soit formulée clairement la thèse de l'existence d'une organisation prégénitale dominée par l'érotisme anal. En 1915, dans la troisième édition des Trois essais, Freud l'intègre au chapitre qui porte sur les «phases de développement de l'organisation sexuelle». Il y distingue explicitement le stade oral et le stade anal. Par la suite, dans l'édition de 1924 des Trois essais, Freud adoptera la subdivision, proposée par Karl Abraham, de ces deux stades prégénitaux, en une phase précoce et une phase tardive. Enfin Freud introduira un

16 Ferenczi 1924, $98 \mathrm{f}$.

17 Sulloway 1992,247-250.

18 Freud 1997a.

19 Freud 1986.

20 Freud 1997b.

21 Freud 1997c. 
dernier stade, le stade phallique ou génital, qui domine dans la sexualité adulte.

Freud, dans le chapitre en question, note que ces phases «évoquent un retour à des états primitifs de la vie animale» ${ }^{22}$. A la même époque, Freud rédige en vue de la publication une observation clinique, L'homme aux loups. Il y reprend l'idée que les phases du développement de l'organisation sexuelle trouvent leur origine dans l'histoire du développement des espèces. «On ne peut s'empêcher», écrit-il, «de faire ici des parallèles biologiques et d'édifier l'hypothèse d'après laquelle les organisations prégénitales de l'homme seraient les vestiges de conditions qui, dans certains classes d'animaux, ont été conservées de façon permanente» ${ }^{23}$. Le premier des Trois essais sur la théorie sexuelle s'intitule «Les aberrations sexuelles». La conception de l'homosexualité qui y est exposée fait écho à celle de Bölsche. Dans une note bas de page de l'édition de 1915, Freud précise son point de vue sur les liens entre analité et homosexualité:

Dans le cas des types inversés, on constate en permanence la prédominance de constitutions archaïques et de mécanismes psychiques primitifs. La valeur accordée aux objets narcissiques et le maintien de la signification érotique de la zone anale paraissent en constituer les caractères les plus essentiels. ${ }^{24}$

Cette fixation ou régression anale fait l'objet de développements en 1913 dans «La disposition à la névrose obsessionnelle», où Freud écrit:

Le courant passif est alimenté par l'érotisme anal, dont la zone érogène correspond à l'ancien cloaque indifférencié. L'accentuation de cet érotisme anal au stade de l'organisation prégénitale déposera chez l'homme quand le stade suivant de la fonction sexuelle, celui des organes génitaux, sera atteint, une importante prédisposition à l'homosexualité..$^{25}$

Freud, d'autre part, étend l'influence de ces restes historiques au-delà du comportement strictement sexuel:

Pour certains traits de caractère, il a même été possible d'établir un lien avec des composantes érogènes déterminées. Ainsi, l'entêtement, l'économie, le goût de l'ordre, découlentils de l'utilisation de l'érotisme anal. L'orgueil est déterminé par une forte disposition à l'érotisme urinaire. ${ }^{26}$

Enfin à propos des théories de la naissance échafaudées par les enfants, qui supposent que les bébés viennent au monde de la même manière que l'on évacue les selles, il note que «ces théories enfantines rappellent certaines dispositions du règne animal, en particulier le cloaque des espèces inférieures aux mammifères $»^{27}$. Il semble donc que Freud voie dans les dif-

22 Freud 1997a, 129.

23 Freud 1992, 409.

24 Freud 1997a, 52.

25 Freud 1997c, 194.

26 Freud 1997c, 190.

27 Freud 1997a, 126. 
férentes composantes de l'érotisme la source même de la complexité du psychisme humain. Dès lors, «la conséquence inévitable de ces considérations est qu'il faut prêter à chaque individu un érotisme oral-anal-urinaire [...] $\gg^{28}$. Toutes ces références soulignent le soubassement évolutionniste des Trois essais. Il est en outre évident qu'il existe une certaine parenté de thème et de terme entre Freud et Bölsche.

Ilse Grubrich Simitis a retrouvé en 1983, joint à une lettre de Freud à Ferenczi, un manuscrit datant de 1915, qui a été publié depuis, et qui est intitulé, Vue d'ensemble sur les névroses de transfert ${ }^{29}$. Dans ce texte, Freud s'appuie sur l'idée suivante: les pathologies du fonctionnement psychique seraient liées à des phénomènes de régression, à des points de fixation de la libido. Sur le manuscrit, à l'attention de Ferenczi, figurait la mention: «Vous pouvez le jeter ou le conserver», ce qui porte à croire que ce que Freud présentait lui-même comme une «fantaisie scientifique» ne répondait pas aux exigences de scientificité qu'il s'était fixées. Dans cette Vue d'ensemble, il se propose d'étudier l'histoire du développement psychique de l'être humain. Pour cela, il dégage deux axes: le développement de la vie sexuelle humaine, c'est-à-dire le développement de la libido, et le développement du moi:

[O]n a l'impression que l'histoire du développement de la libido répète une séquence beaucoup plus ancienne du développement (phylogénétique) que ne le fait l'histoire du développement du moi: la première répète peut-être les conditions de développement de l'embranchement des vertébrés, tandis que la seconde est dépendante de l'histoire de l'espèce humaine. ${ }^{30}$

Freud reprend à la lettre la loi biogénétique de Haeckel, selon laquelle notre développement récapitule l'histoire de nos ancêtres. Les points de fixation des névroses sont dès lors aussi les restes d'acquisitions phylogénétiques, c'est-à-dire en quelque sorte des legs d'événements incontournables du passé. Mais, à vrai dire, Freud s'inspirait de considérations de celui à qui il confia le destin du manuscrit, Ferenczi. «Sans doute réussira-t-on un jour», écrivait ce dernier en 1913, «à mettre en parallèle les différents stades évolutifs du moi, ainsi que leurs types de régressions névrotiques, et les étapes parcourues par l'histoire de l'espèce humaine» ${ }^{31}$. On doit en outre citer les travaux du psychologue américain G. Stanley Hall qui, toujours dans la perspective de Haeckel, voit dans la pathologie mentale la persistance de formes primitives de conduite ${ }^{32}$. Ainsi, Freud, à son tour, dans Vue d'ensemble sur les névroses de transfert, tente d'établir un parallèle entre les psycho-

28 Freud 1997a, 139.

29 Freud 1986.

30 Freud 1986, 30f.

31 Ferenczi 1978, 63.

32 Hall 1904. 
névroses et l'évolution phylogénétique: à chaque entité nosographique, correspondrait une période de «dénuement» de l'histoire de l'humanité. Le bouleversement des circonstances aurait ainsi entraîné l'inscription d'une marque indélébile, une disposition, dans la phylogenèse. Par effet de la loi de Haeckel, ces dispositions se seraient potentialisées en fixation dans le développement ontogénétique. La névrose constituerait donc une régression à ces points de fixation, et les symptômes névrotiques seraient une lutte contre l'instauration nouvelle du «dénuement» vécu par les ancêtres.

Sur leur versant biologique, les écrits spéculatifs de Freud, comme les Trois essais sur la théorie sexuelle, et la Vue d'ensemble sur les névroses de transfert s'avèrent donc nourris d'une pensée évolutionniste dont le livre de Bölsche, Das Liebesleben in der Natur, devait représenter une vulgarisation particulièrement bienvenue, puisque le thème de l'ouvrage est l'évolution de l'amour. Notons enfin, que malgré ces éléments quelque peu troublants, il n'existe pas de travaux portant sur Freud et Bölsche, et les spécialistes de Bölsche n'ont pas non plus rapproché sa vision de la sexualité de celle de la psychanalyse freudienne. La thèse de Rolf Schmidt ${ }^{33}$, qui porte sur l'approche bolschéenne de la sexualité, ne mentionne pas une seule fois le nom de Freud.

La mention du «roman scientifique bolschéen» par le fondateur de la psychanalyse à l'intention d'un auditoire supposé profane en la matière souligne l'importance des sources évolutionnistes de la pensée freudienne et la nécessité de l'éclairage qu'apporte l'histoire des idées. Il ne s'agit évidemment pas de d'affirmer que l'ensemble de l'œuvre de Freud se trouve déjà chez Bölsche, mais plutôt, de souligner l'importance de certains aspects, aujourd'hui sous-estimés ou méconnus, de la culture évolutionniste allemande scientifique et populaire du début du $\mathrm{XX}^{\mathrm{e}}$ siècle. Le style de Bölsche ne semble cependant pas toujours avoir bonne presse auprès des psychiatres qui s'intéressent à la sexologie, comme Forel. Bölsche a cependant le soutien de Haeckel et, surtout, connaît un très large succès populaire. L'évolutionnisme de Freud semble donc puiser ses sources autant dans les écrits scientifiques contemporains des évolutionnistes et des sexologues que dans des textes de vulgarisation. L'historien qui s'intéresse aux rapports qu'entretiennent psychanalyse et évolutionnisme ne saurait se contenter de lire ceux que l'on considère aujourd'hui comme les «grands auteurs», c'est-à-dire Darwin et Lamarck. Il lui faut se réapproprier le climat évolutionniste qui caractérisait la culture allemande au début du $\mathrm{XX}^{\mathrm{e}}$ siècle, ce qui inclut l'étude de vulgarisateurs scientifiques.

33 Schmidt 1964. 


\section{Bibliographie}

Bölsche, Wilhelm, Das Liebesleben in der Natur: eine Entwicklungsgeschichte der Liebe, 3 Vol. (Jena 1898-1903)

- Die naturwissenschaftlichen Grundlagen der Poesie: Prolegomena einer realistischen Äesthetik (Leipzig 1887)

- Entwicklungsgeschichte der Natur, 2 Vol. (Berlin 1894-1896)

Daum, Andreas, «Un pays sans tradition de science populaire?», dans: Bernadette BensaudeVincent (éd.), La science populaire dans la presse et l'édition XIX et XX siècle (Paris 1997) $128-145$

Ellenberger, Henri F., Histoire de la découverte de l'inconscient (Paris 1994)

Federn, Ernst/Herman Nünberg (éds), Les premiers psychanalystes, minutes de la Société psychanalytique de Vienne, vol. 2 1908-1910 (Paris 1978)

Fellman, F., «Ein Zeuge der ästhetischen Kultur im 19. Jahrhundert: Wilhem Bölsche», Archiv für Kulturgeschichte 70 (1988) 131-148

Ferenczi, Sandor, «Le développement du sens de réalité et ses stades», dans: Sandor Ferenczi, Psychanalyse II (Paris 1978) 51-65 [or. 1913]

- Thalassa (Paris 1992) [or. 1924]

Forel, Auguste, La question sexuelle exposée aux adultes cultivés (Paris 1906)

Freud, Sigmund, Introduction à la psychanalyse (Paris 1922) [or. 1917]

- Vue d'ensemble sur les névroses de transfert (Paris 1986) [or. 1915]

- «L'homme aux loups», dans: Sigmund Freud, Cinq psychanalyses (Paris 1992) 325-420 [or. 1918]

- Trois essais sur la théorie sexuelle (Paris 1997a) [or. 1905]

- «Caractère et érotisme anal», dans: Sigmund Freud, Névrose, psychose et perversion (Paris 1997b) 143-148 [or. 1908]

- «La disposition à la névrose obsessionnelle», dans: Sigmund Freud, Névrose psychose et perversion (Paris 1997c) 189-198 [or. 1913]

Hall, G. Stanley, Adolescence: Its Psychology and Its Relations to Physiology, Anthropology, Sociology, Sex, Crime, Religion, Education, 2 Vol. (New York 1904)

Kelly, Alfred, The Descent of Darwin, the Popularization of Darwinism in Germany 1860-1914 (Chapel Hill 1981)

Mengal, Paul (éd.), Histoire du concept de récapitulation: ontogenèse et phylogenèse en biologie et sciences humaines (Paris 1993)

Nöthlich, Rosemarie, Ernst Haeckel - Wilhelm Bölsche. Briefwechsel 1887-1919 (Jena 2002)

Ritvo, Lucille B., L'ascendant de Darwin sur Freud (Paris 1992)

Schmidt, Rolf, Die Auffassung der Sinnlichkeit und die Einstellung zur Sexualität bei Wilhelm Bölsche [thèse de médecine] (Institut für Medizingeschichte der Universität München 1964)

Sulloway, Frank J., Freud, biologiste de l'esprit (Paris 1992) 